SUMMARY. Thrombolytic therapy has gained widespread acceptance as a means of treating coronary artery thrombosis in patients with acute myocardial infarction. Although experimental data have demonstrated that timely reperfusion limits the extent of infarction caused by regional ischemia, there is growing evidence that reperfusion is associated with an inflammatory response to ischemia that exacerbates the tissue injury. Ischemic myocardium releases archidonate and complement-derived chemotactic factors, e.g., leukotriene $B_{4}$ and $C_{5 a}$, which attract and activate neutrophils. Reperfusion of ischemic myocardium accelerates the influx of neutrophils, which release reactive oxygen products, such as superoxide anion and hydrogen peroxide, resulting in the formation of a hydroxyl radical and hypochlorous acid. The latter two species may damage viable endothelial cells and myocytes via the peroxidation of lipids and oxidation of protein sulfhydryl groups, leading to perturbations of membrane permeability and enzyme function. Neutrophil depletion by antiserum and inhibition of neutrophil function by drugs, e.g., ibuprofen, prostaglandins (prostacyclin and $\mathrm{PGE}_{1}$ ), or a monoclonal antibody, to the adherence-promoting glycoprotein Mo-1 receptor, have been shown to limit the extent of canine myocardial injury due to coronary artery occlusion/ reperfusion. Recent studies have challenged the hypothesis that xanthine-oxidase-derived oxygen radicals are a cause of reperfusion injury. Treatment with allopurinol or oxypurinol may exert beneficial effects on ischemic myocardium that are unrelated to the inhibition of xanthine oxidase. Furthermore, the human heart may lack xanthine oxidase activity. Further basic research is needed, therefore, to clarify the importance of xanthine oxidase in the pathophysiology of reperfusion injury. Current data are highly suggestive of a deleterious role of the neutrophil in organ reperfusion and justify consideration of the clinical investigation of neutrophil inhibitors in patients receiving thrombolytic agents during the evolution of an acute myocardial infarction.

KEY WORDS. myocardial ischemia, myocardial infarction, reperfusion injury, oxygen free radicals, neutrophils, xanthine oxidase

Recognition that coronary thrombosis is the precipitating event in the onset of acute myocardial infarction provided the rationale for exploring both pharmacologic and mechanical methods of restoring coronary artery patency [1]. Based on recent multicenter trials, which have demonstrated a reduction of mortality associated with the administration of thrombolytic agents, thrombolytic therapy has become the standard of care for the treatment of acute myocardial infarction [2].
Steven W. Werns, ${ }^{1}$ Benedict R. Lucchesi ${ }^{2}$

${ }^{1}$ Department of Internal Medicine (Division of Cardiology); ${ }^{2}$ Department of Pharmacology, The University of Michigan Medical School, Ann Arbor, Michigan $\mathbf{4 8 1 0 9}$

The knowledge that persistent myocardial ischemia leads to a time-dependent loss of viable myocardial tissue suggests that the early restoration of myocardial blood flow is essential in order to arrest the progression of myocardial cell death and to permit the functional recovery of reversibly injured myocardium. Reperfusion and reoxygenation of the ischemic myocardium, while essential for ultimate tissue survival, may be associated with the risk of extending the area of myocardial injury beyond that which has occurred as a result of the ischemic process. The reintroduction of oxygenated blood to the previously ischemic myocardial tissue may be detrimental to the survival of the reoxygenated cell, as well as beneficial. A major effort has been expended in recent years to gain a better understanding of the phenomena that have been referred to as the oxygen paradox [3-5] and reperfusion injury $[5,6]$. The suggestion has been made that oxygen radicals are involved in both processes [4-6]. Extensive research has been devoted toward reducing the extent of tissue injury associated with ischemia and reperfusion by modifying the sequence of events that are thought to be involved in the generation of oxygen free radicals. Several authors have reviewed recent studies that support the concept of oxygen-radical-mediated reperfusion injury [5-9]. This review will focus on the role of oxygen free radicals in the extension of cardiac injury during reperfusion after regional myocardial ischemia and, in particular, on the important role of the polymorphonuclear leukocyte as a primary mediator of reperfusion injury due to its capacity to generate reactive species of oxygen.

This study was supported by the National Institutes of Health, Heart, Lung and Blood Institute, Grant \#19782-06 and by a Grant-in-Aid from the American Heart Association of Michigan. Dr. Werns is the recipient of a Physician Scientist Award from the National Institutes of Health, Heart, Lung and Blood Institute, Grant \#HL-01409-01. Address for correspondence and reprint requests: Benedict $R$. Lucchesi, $\mathrm{PhD}, \mathrm{MD}$, The University of Michigan Medical School, Department of Pharmacology, 6322 Medical Science Building I, Ann Arbor, Michigan 48109-0626, Tel. (313) 764-9116. 


\section{Oxidative Stress}

All mammalian cells are subjected to free radical reactions that occur continuously in vivo as a result of both enzymatic and nonenzymatic mechanisms, although the former is most likely the more important reactive process. Oxidative stress occurs in cells and tissues when there is an increase in the rate of generation of superoxide anion $\left(\mathrm{O}_{2}^{-}\right)$and hydrogen peroxide $\left(\mathrm{H}_{2} \mathrm{O}_{2}\right)$ that exceeds the capacity of the endogenous cellular defenses. Enhanced production of $\mathrm{O}_{2}^{-}$can occur in tissues as a result of an increase in the oxygen tension, the presence of toxic substances that increase intracellular oxidant formation (e.g., adriamycin, acetominophen, paraquat), or as a result of the activation of specific enzymatic mechanisms that are capable of leading to the formation of $\mathrm{O}_{2}^{-}$(NADPH-oxidase in neutrophils or xanthine oxidase) $[8,9]$. Thus, the accumulation of inflammatory cells at a site of ischemic injury may contribute to an extension of the cellular destruction by mechanisms unrelated to oxygen deprivation.

$\mathrm{H}_{2} \mathrm{O}_{2}$ exerts an oxidant stress by virtue of its conversion via a metal ion-dependent reaction to the hydroxyl radical $(\mathrm{OH} \cdot)$. The latter reaction is enhanced by the presence of $\mathrm{O}_{2}^{-}$. The extreme reactivity of $\mathrm{OH} \cdot$ limits its interaction with biomolecules to diffusion controlled reactions, so that the reactive species of oxygen must interact with its target close to the site at which it is formed. In this instance, iron ions are considered to be the most likely promoters of $\mathrm{OH} \cdot$ ion formation [ $[10$, 11]. The source of iron may come from ferritin when it reacts with $\mathrm{O}_{2}^{-}[12]$ and/or from hemoglobin, which is degraded in the presence of $\mathrm{H}_{2} \mathrm{O}_{2}$ [13].

\section{Antioxidant Mechanisms}

Aerobic cells have the capacity to remove the reactive products of oxygen that are formed during normal metabolic events. The primary intracellular defense mechanisms against oxidative stress include superoxide dismutase, glutathione peroxidase, catalase, and vitamin $E$ localized in the lipid membrane. Superoxide dismutase is present in the mitochondria of mammalian cells as manganese superoxide dismutase and in the cytosol as a copper-zinc form of the enzyme. The enzyme catalzyes the following reaction:

$$
2 \mathrm{O}_{2}^{-}+2 \mathrm{H}^{+} \rightarrow \mathrm{O}_{2}+\mathrm{H}_{2} \mathrm{O}_{2} .
$$

The rate constant for the reaction shown above is suf- ficiently rapid so that little if any superoxide anion is available to react with the hydrogen peroxide, which might otherwise lead to the formation of hydroxyl anion via the metal-catalyzed Haber-Weiss reaction shown below:

$$
\begin{aligned}
\mathrm{H}_{2} \mathrm{O}_{2}+\mathrm{Fe}^{2+} & \rightarrow \mathrm{OH} \cdot+\mathrm{OH}^{-}+\mathrm{Fe}^{3+} \\
\mathrm{O}_{2}^{-}+\mathrm{Fe}^{3+} & \rightarrow \mathrm{O}_{2}+\mathrm{Fe}^{2+} .
\end{aligned}
$$

The decomposition of hydrogen peroxide to water and oxygen can be catalyzed by the enzymes catalase and glutathione peroxidase. The latter enzyme also catalyzes the reduction of lipid peroxides. The reduction of hydrogen peroxide or lipid peroxides by glutathione peroxidase is accompanied by the oxidation of glutathione, resulting in the formation of glutathione disfulfide. Glutathione is regenerated from glutathione disfulfide by glutathione reductase; but under conditions of excessive oxidative stress, regeneration of glutathione may be inadequate to prevent the peroxidation of membrane lipids [14].

Whereas the intracellular compartment contains several antioxidant mechanisms, the main extracellular defense against free radicals resides with the copper-containing protein, ceruloplasmin [8]. Several studies, however, have demonstrated that erythrocytes may remove extracellular hydrogen peroxide, thereby protecting surrounding tissue against damage mediated by hydrogen peroxide or its secondary products, hydroxyl anion and hypochlorous acid [15-17]. The latter oxidant is produced by neutrophil-derived myeloperoxidase. Erythrocytes were shown to prevent the oxidation of cytochrome $\mathrm{C}$, damage to cultured endothelial cells, and lung edema caused by exogenous hydrogen peroxide [15]. The inhibition of erythrocyte catalase abolished the protection afforded by erythrocytes, and the addition of purified catalase restored protection [16]. Erythrocytes were unable to inhibit the oxidation of cytochrome $\mathrm{C}$ by superoxide anions [17], and the inhibition of erythrocyte superoxide dismutase did not affect the ability of erythrocytes to prevent xanthine-oxidase-induced injury of target cells [16]. Thus, erythrocytes would be expected to provide other cells or tissues protection from hydrogen peroxide, but not from superoxide anions generated in their environment [17]. The fact that plasma and/or blood may provide an effective antioxidant defense against the presence of extracellular hydrogen peroxide but not superoxide anions suggests that the exogenous addition of superoxide dismutase could modulate reactions of the free radical species of oxygen, while the addition of catalase may not. This has been suggested by the observation that superoxide dismutase alone was found 
to limit ischemic myocardial injury in vivo, whereas catalase was without benefit [18].

\section{Myocardial Ischemia and Leukocytes}

Myocardial infarction is associated with an inflammatory response that has been regarded previously as a repair process that replaces necrotic tissue with a collagenous scar. Recently, there has been growing recognition that the injury associated with the infiltration of polymorphonuclear leukocytes during ischemia, and especially upon reperfusion, could be additive to that resulting from the ischemic process itself. The infiltration of ischemic canine myocardium by neutrophils begins within 60 minutes of coronary occlusion and is enhanced significantly by reperfusion $[19,20]$.

The hypothesis that neutrophils contribute to the extension of ischemic damage and participate in reperfusion injury is supported by numerous studies that have demonstrated that agents that inhibit neutrophil function or interfere with the products of neutrophils limit myocardial injury in experimental animals subjected to coronary artery occlusion followed by reperfusion [7]. Romson et al. [21] examined the effect of an antineutrophil antiserum on the extent of canine myocardial injury due to regional ischemia for 90 minutes followed by reperfusion for 6 hours. Dogs treated with the antiserum showed a $77 \%$ reduction in the blood leukocyte count and $43 \%$ smaller infarcts compared to dogs treated with a nonimmune serum. There were no hemodynamic effects of the antiserum to account for the reduction of infarct size, indicating that the limitation of myocardial injury was related to the neutropenic state. Subsequently, Mullane et al. [22] observed that a $60 \%$ reduction in the circulating neutrophil count induced by treatment with hydroxyurea resulted in a significant decrease in canine myocardial injury due to coronary artery occlusion and reperfusion.

Drugs that inhibit neutrophil function without causing neutropenia have also been shown to be cardioprotective during experimental regional myocardial ischemia and reperfusion. Romson et al. [23] demonstrated that the reduction of canine myocardial infarct size by ibuprofen, for example, was associated with a decreased accumulation of neutrophils in the reperfused ischemic myocardium. Flynn et al. [24] studied the effects of ibuprofen and aspirin on feline neutrophil function and myocardial infarction. Ibuprofen, but not aspirin, inhibited the respiratory burst of activated neutrophils and reduced the extent of myocardial damage. Thus, the cardioprotective effects of the nonsteroidal antiinflammatory agents are related to the inhibition of neutrophil function, rather than the inhibition of the cyclooxygenase pathway.

Simpson and colleagues [25-27] have investigated the mechanism by which prostaglandin $\mathrm{E}_{1}\left(\mathrm{PGE}_{1}\right)$, prostacyclin $\left(\mathrm{PGI}_{2}\right)$, and iloprost, a stable analog of $\mathrm{PGI}_{2}$, salvage reperfused ischemic myocardium. Each agent was found to inhibit the generation of superoxide anions by activated neutrophils in vitro and to limit canine myocardial injury caused by coronary artery occlusion and reperfusion in vivo. The compound SC39902, another stable analog of $\mathrm{PGI}_{2}$, exerted hemodynamic effects similar to $\mathrm{PGI}_{2}$, but did not reduce infarct size or inhibit neutrophil function [25], suggesting that $\mathbf{P G I}_{2}, \mathbf{P G I}_{1}$, and iloprost limit reperfusion injury by suppressing neutrophil-mediated tissue damage.

\section{Complement and Leukotriene $\mathrm{B}_{4}$}

The complement system serves a major role in the production of mediators involved in the acute inflammatory response. Chemotactic factors that are generated by the activation of the complement system, e.g., $\mathrm{C}_{3 \mathrm{a}}$, participate in the recruitment and activation of neutrophils, whereas the anaphylatoxins, e.g., $\mathrm{C}_{5 \mathrm{a}}$, mediate changes in vascular permeability. Enzymes may react with individual complement components, resulting in the formation of inflammatory mediators, without causing generalized activation of the complement cascade [28, 29]. Hill and Ward [28, 29] demonstrated the presence of a tissue protease in ischemic myocardium that cleaves the third component of complement into chemotactically active fragments that may stimulate the attraction of neutrophils to the myocardium. Other investigators have focused attention on the ability of mitochondria and cardiac subcellular membranes to activate the complement system [30-33]. Rossen and coworkers [33] have presented evidence that the cardiac lympatic drainage during postischemic reperfusion contains molecules of cardiac subcellular origin that are bound to Clq. The authors postulate that the formation of macromolecular complexes may be one of the stimuli that provokes the inflammatory response to myocardial ischemia. The concept that myocardial ischemia is associated with complement-induced injury that is mediated by neutrophils is supported by experiments which showed that depletion of complement by treatment with cobra venom factor limits both the influx of neutrophils and the release of creatine kinase by ischemic myocardium [34]. 
Neutrophils trapped within the vascular capillary bed, as well as those that have extravasated into the extravascular space, are capable of attracting and activating additional phagocytic cells through their ability to synthesize lipoxygenase metabolites of arachidonic acid, including leukotriene $\mathrm{B}_{4}\left(\mathrm{LTB}_{4}\right)$, one of the most potent neutrophil chemoattractants. $\mathrm{LTB}_{4}$ amplifies the neutrophil-mediated injury by stimulating the chemotaxis of neutrophils and their release of reactive oxygen species. The tissue concentration of $\mathrm{LTB}_{4}$ and other eicosanoids is greatly increased in myocardium that has undergone regional ischemia [22, 35], and in one study the peak concentration of $\mathrm{LTB}_{4}$ preceded the peak influx of neutrophils, suggesting that the myocardium itself may produce $\mathrm{LTB}_{4}$ during ischemia [35]. Drugs that inhibit the 5-lipoxygenase pathway, such as BW 755C and nafazatrom, have been shown to reduce neutrophil infiltration and tissue damage of the ischemic/reperfused myocardium [22, $36,37]$. BW $755 \mathrm{C}$ and nafazatrom, however, display multiple pharmacologic effects, and the study of pure lipoxygenase inhibitors and leukotriene antagonists will be necessary to clarify the importance of leukotriene-mediated events during myocardial ischemia and reperfusion.

\section{Adherence-Promoting Cell-Surface Glycoprotein Complexes}

Leukocyte attachment to target cells may be a prerequisite for neutrophil-mediated cellular injury. Leukocytes have cell-surface glycoprotein complexes, referred to as the CDw18 complex or LFA antigens, that are involved in cell-cell and cell-surface interactions [38-40]. The adherence molecules are heterodimers, consisting of common beta subunits with different alpha subunits that dictate the molecular specificity. The Mo-1 complex, a heterodimer present on both human and animal (canine, nonhuman primates) phagocytic cells, is functionally very similar, or may be identical, to the $\mathrm{C} 3 \mathrm{bi}$ receptor (CR3), which mediates adherence to cells coated with $\mathrm{C} 3 \mathrm{bi}$. Activation of neutrophils increases the expression of the adhesion-promoting receptors [41]. Using monoclonal antibodies directed against the Mo-1 glycoprotein complex, it was demonstrated that the Mo- 1 receptor is responsible for the attachment of neutrophils to a variety of substrates, including vascular endothelium and pulmonary alveolar epithelium [42]. An anti-Mo-1 monoclonal antibody not only prevented the adherence of neutrophils to pulmonary alveolar epithelial cells, but reduced the extent of cell injury caused by the neutrophils [42].

The same anti-Mo-1 monoclonal antibody was used to further investigate the contribution of neutrophils to myocardial injury caused by ischemia and reperfusion. Administration of the anti-Mo-1 antibody 45 minutes after the induction of regional myocardial ischemia significantly reduced the extent of myocardial injury in dogs subjected to coronary artery occlusion for 90 minutes, followed by reperfusion for 6 hours [43]. The antibody had no effect on arterial blood pressure, heart rate, or coronary artery blood flow, which could account for the observed protective effect. The results indicate that neutrophil adhesive interactions are an important step in neutrophil-mediated myocardial injury and that the extent of myocardial damage may be reduced by inhibiting neutrophil adhesive interactions. These observations provide additional evidence for the important role of inflammatory cells in extending myocardial injury beyond that caused by the ischemic interval itself.

\section{Mediators of Leukocyte-Induced Myocardial Injury}

Neutrophils that accumulate within the reperfused myocardium may exert deleterious effects via several mechanisms. Compared to erythrocytes, neutrophils are less deformable and more susceptible to trapping in the microcirculation during ischemia [44]. Engler et al. [44] observed that $60 \%$ of the capillaries in the region of myocardium subjected to ischemia and reperfusion were obstructed by leukocytes and displayed evidence of the "no reflow" phenomenon. The authors postulated that failure to maintain reflow to the previously ischemic myocardial region was related to the progressive obstruction of the capillary bed by the accumulating neutrophils that adhered to the endothelial surface of the vasculature. Thus, mechanical plugging of capillaries, resulting in the "no reflow" phenomenon, is one mechanism by which neutrophils may exacerbate ischemic myocardial injury.

\section{Proteases}

Once attached to the vascular endothelium, neutrophils are capable of becoming fully activated by complement products, leukotriene $\mathrm{B}_{4}$, or platelet-activating factor, releasing destructive proteases and toxic oxygen products. The human neutrophil contains two latent metalloproteinases, collagenase and gelatinase, 
which are released upon activation of the cell and which are activated by hypochlorous acid. Treatment with aprotinin, a nonspecific inhibitor of proteolysis, limited the extent of canine myocardial infarction, suggesting that lysosomal proteases may participate in the progressive destruction of otherwise viable myocardial tissue [45]. Aprotinin also exerts a direct inhibitory effect on neutrophils, however, that may account for its cardioprotective property [46]. Bolli et al. [47] were unable to confirm the observation regarding the deleterious role of proteolytic lysosomal enzymes as mediators of ischemic myocardial injury. Thus, the relationship between lysosomal enzymes and ischemic myocardial injury remains unsettled and requires further assessment.

\section{Oxygen Radicals}

The activation of neutrophils by chemotactic factors, such as $\mathrm{C}_{5 \mathrm{a}}$, platelet activating factor, or leukotriene $\mathrm{B}_{4}$, stimulates the NADPH oxidase of the neutrophil cell membrane to catalyze the univalent reduction of oxygen to yield superoxide anion, an oxygen free radical capable of giving rise to other activated products of oxygen such as hydrogen peroxide and hydroxyl anion. The latter may be derived from the interaction of hydrogen peroxide with lactoferrin, another product released by activated neutrophils [48]. Neutrophilderived myelperoxidase catalyzes the reaction of hydrogen peroxide with chloride anions, forming hypochlorous acid, a highly reactive oxidant [49]. The oxygen metabolites released by activated neutrophils have been shown to cause peroxidation of membrane lipids, denaturation of proteins, and degradation of interstitial matrix molecules, resulting in altered membrane permeability and enzymatic activity [50].

Numerous studies have been conducted to investigate the effects of oxygen radical scavengers on experimental myocardial injury due to regional or global ischemia. Jolly et al. [51] examined the effect of concomitant treatment with superoxide dismutase (SOD), an enzyme that dismutates superoxide anion, and catalase, an enzyme that degrades hydrogen peroxide on myocardial injury in dogs undergoing occlusion of the left circumflex coronary artery for 90 minutes, followed by reperfusion for 24 hours. Infusions of SOD in combination with catalase, started either 15 minutes before coronary occlusion or 15 minutes before reperfusion, were equally effective in reducing myocardial tissue damage, suggesting that oxygen-radical-mediated injury is temporally related to the onset of reperfusion. Subsequently, treatment with SOD alone was compared to treatment with catalase alone using the same experimental preparation [18]. Dogs receiving SOD alone had $50 \%$ smaller infarcts than control dogs, while catalase had no significant beneficial effect on infarct size, suggesting that reperfused ischemic myocardium is susceptible to superoxide-anion-mediated damage. The failure of catalase to reduce myocardial injury may relate to the abundant catalase activity of erythrocytes, which are capable of detoxifying hydrogen peroxide, but not superoxide anions, produced in the local environment [17]. Thus, blood-perfused systems subjected to ischemia and reperfusion may be protected from hydrogen peroxide, but unable to detoxify superoxide anions due to the relative absence of protective mechanisms in the interstitial space where invading leukocytes react with the surrounding tissues and release cytotoxic oxygen intermediates.

The action of SOD is presumed to occur at sites of superoxide anion radical formation, where neutrophils adhere to endothelial cell membranes or extravasate into the extravascular space. Treatment with SOD that was delayed until 40 minutes after coronary artery reperfusion, however, did not reduce myocardial injury, suggesting that the cellular damage produced by oxygen radicals occurs upon, or soon after, reperfusion-perhaps before the peak accumulation of neutrophils exiting from the vascular compartment into the interstitial space [51]. Furthermore, recent studies have arrived at conflicting conclusions regarding the ability of SOD to limit the extent of experimental myocardial infarction. Werns et al. [52] and Ambrosio et al. [53] reported that treatment with SOD reduced infarct size in dogs subjected to coronary artery occlusion for 90 minutes followed by reperfusion for 24 hours [52] or 48 hours [53]. When the extent of myocardial injury due to 90 minutes of ischemia was assessed after 4 days [54] or 7 days [55] of reperfusion, a beneficial effect of treatment with "native" SOD was not demonstrable, but treatment with PEG-SOD, which has a prolonged plasma half-life due to the polyethylene glycol conjugate, was found to significantly reduce the extent of infarction after coronary artery occlusion for 90 minutes, followed by reperfusion for 7 days [56]. The latter study implies that effective therapy directed against damage caused by oxygen radicals requires an agent with a prolonged duration of action. Similarly, Simpson et al. [57] found that sustained limitation of myocardial injury by treatment with iloprost, a prostacyclin analogue that inhibits neutrophil function, requires treatment extending beyond the first several hours after coronary artery reperfusion. 


\section{The Role of Xanthine Oxidase in Myocardial Reperfusion Injury}

The enzyme xanthine oxidase is an additional proposed source of oxygen radicals within reperfused ischemic myocardium. Chambers et al. [58] reported that myocardial ischemia causes the conversion of myocardial xanthine dehydrogenase, which does not utilize oxygen as a substrate, to xanthine oxidase, which can reduce oxygen to superoxide anion and hydrogen peroxide. They hypothesized that xanthine oxidase utilizes hypoxanthine and xanthine as substrates to generate superoxide anions during reperfusion of ischemic myocardium. Dogs treated with allopurinol, an inhibitor of xanthine oxidase, beginning 1 day before temporary coronary artery occlusion, had significantly smaller infarcts than control dogs, supporting the hypothesis. Using a dosing protocol similar to that employed by Chambers et al. [58], experiments performed in this laboratory demonstrated a $40 \%$ reduction of myocardial injury after coronary artery occlusion for 90 minutes, followed by reperfusion for 6 hours [59].

Additional studies have contradicted the conclusion that xanthine oxidase inhibition reduces the extent of regional myocardial injury in the dog. Reimer and Jennings [60] did not observe a favorable effect of allopurinol in dogs subjected to coronary artery occlusion for 40 minutes, followed by reperfusion for 4 days. The latter study differed from previous studies because allopurinol treatment did not commence until 30 minutes before coronary artery occlusion. Subsequently, a study performed by the authors showed that treatment with allopurinol beginning 15 minutes before reperfusion failed to limit myocardial injury in dogs subjected to 90 minutes of coronary artery occlusion followed by 6 hours of reperfusion [61]. Oxypurinol, a metabolite of allopurinol that has a prolonged plasma half-life and acts as a noncompetitive inhibitor of xanthine oxidase, significantly reduced the extent of injury when the drug was administered both 15 minutes before reperfusion and 3 hours after reperfusion [61]. Several additional laboratories have examined the effect of oxypurinol on canine myocardial infarction [62-64]. Studies conducted by Matsuki et al. [62] and Puett et al. [63] employed a protocol consisting of coronary artery occlusion for 90 minutes followed by reperfusion for 24 hours. Treatment with either allopurinol or oxypurinol administered as a bolus of $10 \mathrm{mg} / \mathrm{kg} 15$ minutes before reperfusion, followed by an infusion of $55 \mathrm{mg} / \mathrm{kg} /$ day for 1 day, caused a marked and similar limitation of infarct size [62]. When oxypurinol therapy was limited to a $25-\mathrm{mg} /$ $\mathrm{kg}$ dose 30 minutes before reperfusion, there was no detectable effect on the extent of infarction [63]. Similarly, the administration of oxypurinol, $10 \mathrm{mg} / \mathrm{kg}$, 10 minutes before occlusion and 10 minutes before reperfusion, failed to alter the size of infarction in dogs subjected to coronary artery occlusion for $\mathbf{4 0}$ minutes and reperfusion for 4 days [64]. Thus, the data regarding the effects of both allopurinol and oxypurinol on canine infarct size are conflicting, perhaps due to the different schedules of drug administration. Treatment with oxypurinol that ceased at the time of reperfusion was ineffective $[63,64]$, while therapy that was maintained throughout reperfusion until the time of sacrifice significantly reduced the extent of injury $[61,62]$. Analagous results were obtained in dogs treated with iloprost, a prostacylin analog [57].

Although experiments performed in our laboratory found that the administration of allopurinol $15 \mathrm{~min}$ utes before reperfusion did not reduce the extent of myocardial injury, the efflux of uric acid from the coronary sinus during reperfusion was equally suppressed by allopurinol and oxypurinol, suggesting that cardiac xanthine oxidase activity was inhibited [61]. Independent laboratories have reported that xanthine oxidase activity is undetectable in the rabbit heart [6568] or the pig heart [69], although several studies have concluded that allopurinol limited ischemic myocardial injury in the rabbit [66, 70] and pig [69]. Thus, multiple studies suggest that allopurinol and oxypurinol may exert salutory effects on myocardial injury that are unrelated to the inhibition of xanthine oxidase. Peterson et al. [71] have proposed that allopurinol might facilitate electron transfer between the components of the respiratory chain within ischemic mitochondria. Other data indicate that allopurinol and oxypurinol may act as scavengers of reactive oxygen metabolites, including hydroxyl radical and hypochlorous acid $[69,72,73]$. This view is not shared by all investigators, as suggested by a recent study [74] which demonstrated that allopurinol does not produce its beneficial effects by scavenging oxidants produced in the extracellular fluid by activated neutrophils.

No xanthine oxidase activity was detected by biochemical analysis of human myocardium $[75,76]$, although immunohistochemical studies purported to demonstrate the enzyme in the capillary endothelial cells of most organs, including the human heart [77]. Thus, further research is required to clarify the importance of xanthine oxidase in the pathogenesis of postischemic myocardial injury. 


\section{Conclusions}

There are multiple potential sources of oxygen radicals during the reperfusion of ischemic myocardium. There are contradictory data regarding the importance of xanthine-oxidase-derived free radicals in the pathogenesis of reperfusion injury. Abundant evidence suggests that neutrophils attracted to ischemic myocardium by eicosanoids and complement-derived peptides damage viable tissue via the generation of cytotoxic species of oxygen. Thus, agents that are directed against the mechanisms of neutrophil chemotaxis and activation, such as prostacyclin and antiMo-1 antibodies, may be worthy of clinical investigation in patients receiving thrombolytic agents during acute myocardial infarction.

\section{References}

1. DeWood MA, Spores J, Notske R, et al. Prevalence of total coronary occlusion during the early hours of transmural myocardial infarction. $N$ Engl $J$ Med 1980;303:897-902.

2. Topol EJ. Advances in thrombolytic therapy for acute myocardial infarction. J Clin Pharmacol 1987;27:735-745.

3. Hearse DJ, Humphrey SM, Bullock GR. The oxygen paradox and the calcium paradox. Two facets of the same problem? J Mol Cell Cardiol 1978;10:641-648.

4. Guarnieri C, Flamigni F, Caldarera CM. Role of oxygen in the cellular damage induced by re-oxygenation of hypoxic heart. J Mol Cell Cardiol 1980;12:797-808.

5. Hess ML, Manson NH. Molecular oxygen: Friend and foe. The role of the oxygen free radical system in the calcium paradox, the oxygen paradox and ischemia/reperfusion injury. J Mol Cell Cardiol 1984;16:969-985.

6. McCord JM. Oxygen-derived free radicals in post ischemic tissue injury. $N$ Engl $J$ Med 1985;312:159-163.

7. Lucchesi BR, Mullane KM. Leukocytes and ischemiainduced myocardial injury. Ann Rev Pharmacol Toxicol 1986;26:201-204.

8. Southorn PA, Powis G. Free radicals in medicine: I. Chemical nature and biologic reactions. Mayo Clin Proc 1988; 63:381-389.

9. Southorn PA, Powis G. Free radicals in medicine: II. Involvement in human disease. Mayo Clin Proc 1988;63:390-408.

10. Halliwell B, Gutteridge JMC. The importance of free radicals and catalytic metal ions in human diseases. Molec Aspects Med 1985;8:89-193.

11. Halliwell B. Oxidants and human disease: Some new concepts. FASEB $J$ 1987;1:358-364.

12. Biemond P, Van Eijk HG, Swaak AJG, et al. Iron mobilization from ferritin by $\mathrm{O}_{2}$ derived from stimulated polymorphonuclear leukocytes. Possible mechanism in inflammation disease. J Clin Invest 1984;73:1576-1579.

13. Gutteridge JMC. Iron promoters of the Fenton reaction and lipid peroxidation can be released from haemoglobin by peroxides. FEBS Lett 1986;201:291-295.

14. Noronha-Dutra AA, Steen EM. Lipid peroxidation as a mechanism of injury in cardiac myocytes. Lab Invest 1982;47:346-353.

15. Toth KM, Clifford DP, Berger EM, et al. Intact human erythrocytes prevent hydrogen peroxide-mediated damage to isolated perfused rat lungs and cultured bovine pulmonary artery endothelial cells. $J$ Clin Invest 1984;74:292-295.

16. Agar NS, Sadrzadeh SMH, Hallaway PE, et al. Erythrocyte catalase: A somatic oxidant defense. J Clin Invest 1986; $77: 319-321$.

17. Winterbourn CC, Stern A. Human red cells scavenge extracellular hydrogen peroxide and inhibit formation of hypochlorous acid and hydroxyl radical. $J$ Clin Invest 1987;80:1486-1491.

18. Werns SW, Shea MJ, Dirscoll EM, et al. The independent effects of oxygen radical scavengers on canine infarct size. Reduction by superoxide dismutase but not catalase. Circ Res 1985;56:895-898.

19. Rossen RD, Swain JL, Michael LH, et al. Selective accumulation of the first component of complement and leukocytes in ischemic canine heart muscle. A possible initiator of an extramyocardial mechanism of ischemic injury. Circ Res 1985;57:119-130.

20. Chatelain P, Latour J-G, Tran D, et al. Neutrophil accumulation in experimental myocardial infarcts: Relation with extent of injury and effect of reperfusion. Circulation 1987;75:1083-1090.

21. Romson JL, Hook BG, Kunkel SL, et al. Reduction of the extent of ischemic myocardial injury by neutrophil depletion in the dog. Circulation 1983;67:1016-1023.

22. Mullane KM, Read N, Salmon JA, et al. Role for leukocytes in acute myocardial infarction in anesthetized dogs: Relationship to myocardial salvage by antiinflammatory drugs. $J$ Pharmacol Exp Ther 1984;228:510-552.

23. Romson JL, Hook BG, Rigot VH, et al. The effect of ibuprofen on accumulation of indium-111-labeled platelets and leukocytes in experimental myocardial infarction. Circulation 1982;66:1002-1011.

24. Flynn PF, Becker WK, Vercellotti GM, et al. Ibuprofen inhibits granulocyte responses to inflammatory mediators: A proposed mechanism for reduction of experimental myocardial infarct size. Inflammation 1984;8:33-44.

25. Simpson PJ, Mitsos SE, Ventura A, et al. Prostacyclin protects ischemic-reperfused myocardium in the dog by inhibition of neutrophil activation. Am Heart $J$ 1987;113:129137.

26. Simpson PJ, Mickelson JK, Fantone JC, et al. Iloprost inhibits neutrophil function in vitro and in vivo and limits experimental infarct size in canine heart. Circ Res 1987;60:666673.

27. Simpson PJ, Mickelson J, Fantone JC, et al. Reduction of experimental canine myocardial infarct size with prostaglandin E1: Inhibition of neutrophil migration and activation. $J$ Pharmacol Exp Ther 1988;244:619-624.

28. Hill JH, Ward PA. C3 leukotactic factors produced by a tissue protease. $J$ Exp Med 1969;130:505-518.

29. Hill JH, Ward PA. The phlogistic role of C3 leukotactic fragments in myocardial infarcts of rats. $J$ Exp Med 1971;133:885-900.

30. Pinckard RN, Olson MS, Giclas PC, et al. Consumption of classical complement components by heart subcellular membranes in vitro and in patients after acute myocardial infarction. $J$ Clin Invest 1975;56:740-750. 
31. Giclas PC, Pinckard RN, Olson MS. In vitro activation of complement by isolated human heart subcellular membranes. J Immunol 1979;122:146-151.

32. Storrs SB, Kolb WP, Pinckard RN, et al. Characterization of the binding of purified human Clq to heart mitochondrial membranes. $J$ Biol Chem 1981;256:10924-10929.

33. Rossen RD, Michael LH, Kagiyama A, et al. Mechanism of complement activation after coronary artery occlusion: Evidence that myocardial ischemia in dogs causes release of constituents of myocardial subcellular origin that complex with human Clq in vivo. Circ Res 1988;62:572-584.

34. Pinckard RN, O'Rourke RA, Crawford MH, et al. Complement localization and mediation of ischemic injury in baboon myocardium. $J$ Clin Invest 1980;66:1050-1056.

35. Sasaki K, Ueno A, Katori M, et al. Detection of leukotriene $\mathrm{B} 4$ in cardiac tissue and its role in infarct extension through leucocyte migration. Cardiovasc Res 1988;22:142-148.

36. Bednar M, Smith B, Pinto A, et al. Nafazatrom-induced salvage of ischemic myocardium in anesthetized dogs is mediated through inhibition of neutrophil function. Circ Res 1985;57:131-141.

37. Shea MJ, Murtagh JJ, Jolly SR, et al. Beneficial effects of nafazatrom on ischemic reperfused myocardium. Eur $J$ Pharmacol 1984;102:63-70.

38. Anderson DC, Schamalsteig FC, Shearer W, et al. Leukocyte LFA-1, OKM1, p150,95 deficiency syndrome: Functional and biosynthetic studies of three kindreds. Fed Proc 1985;44:2671-2677.

39. Arnaout MA, Dana N, Pitt J, et al. Deficiency of two human leukocyte surface membrane glycoproteins (Mol, and LFA1). Fed Proc 1985;44:2664-2670.

40. Springer TA. The LFA-1, Mac-1 glycoprotein family and its deficiency in an inherited disease. Fed Proc 1985;44:26602663.

41. Berger M, O'Shea J, Cross AS. Human neutrophils increase expression of $\mathrm{C} 3 \mathrm{bi}$ as well as $\mathrm{C} 3 \mathrm{~b}$ receptors upon activation. $J$ Clin Invest 1984;74:1566-1571.

42. Simon RH, DeHart PD, Todd RF. Neutrophil-induced injury of rat pulmonary alveolar epithelial cells. $J$ Cell Invest 1986;78:1375-1386.

43. Simpson PJ, Todd RF, Fantone JC, et al. Reduction of experimental canine myocardial reperfusion injury by a monoclonal antibody (Anti-Mo-1, Anti-CD11b) that inhibits leukocyte adhesion. J Clin Invest 1988;81:624-629.

44. Engler RL, Schmid-Schonbein GW, Pavelec RS. Leukocyte capillary plugging in myocardial ischemia and reperfusion in the dog. Am $J$ Pathol 1983;111:98-111.

45. Hartmann JR, Robinson JA, Gunnar RM. Chemotactic activity in the coronary sinus after experimental myocardial infarction. Effects of pharmacologic interventions on ischemic injury. Am J Cardiol 1977;40:550-555.

46. Hallett MB, Shandall A, Young HL. Mechanism of protection against "reperfusion injury" by aprotinin: Roles of polymorphonuclear leukocytes and oxygen radicals. Biochem Pharmacol 1985;34:1757-1761.

47. Bolli R, Cannon RO, Speir E, et al. Role of cellular proteinases in acute myocardial infarction. II. Influence of in vivo suppression of myocardial proteolysis by antipain, leupeptin and pepstatein on myocardial infarct size in the rat. J Am Coll Cardiol 1983;2:681-688.

48. Ambruso DR, Johnston RB. Lactoferrin enhances hydroxyl radical production by human neutrophils, neutrophil particulate fractions, and an enzymatic generating system. $J$ Clin Invest 1981;67:352-360.
49. Weiss SJ, Klein R, Slivka A, et al. Chlorination of taurine by human neutrophils. J Clin Invest 1982;70:598-607.

50. Fantone JC, Ward PA. Role of oxygen-derived free radicals and metabolites in leukocyte-dependent inflammatory reactions. Am J Path 1982;107:397-418.

51. Jolly SR, Kane WJ, Bailie MB, et al. Canine myocardial reperfusion injury: Its reduction by the combined administration of superoxide dismutase and catalase. Circ Res 1984;54:277-285.

52. Werns SW, Simpson PJ, Mickelson JK, et al. Sustained limitation by superoxide dismutase of canine myocardial injury due to regional ischemia followed by reperfusion. $J$ Cardiovasc Pharmacol 1988;11:36-44.

53. Ambrosio G, Becker LC, Hutchins GM, et al. Reduction in experimental infarct size by recombinant human superoxide dismutase: Insights into the pathophysiology of reperfusion injury. Circulation 1986;74:1424-1433.

54. Richard VJ, Murry CE, Jennings RB, et al. Superoxide dismutase and catalase do not limit infarct size after 90 minutes of ischemia and 4 days of reperfusion in dogs (abstr). Circulation 1987;76:IV-199.

55. Nejima J, Canfield DR, Manders WT, et al. Failure of superoxide dismutase and catalase to alter size of infarct and functional recovery in conscious dogs with reperfusion (abstr). Circulation 1987;76:IV-198.

56. Tamura Y, Driscoll EM, Senyshyn JC, et al. Effects of polyethylene glycol-superoxide dismutase on myocardial infarct size and scar formation in the canine heart (abstr). Circulation 1987;76:IV-200.

57. Simpson PJ, Mickelson J, Fantone JC, et al. Sustained myocardial protection by iloprost with prolonged infusion in a canine model of temporary regional ischemia (abstr). Fed Proc 1987;46:1144.

58. Chambers DE, Parks DA, Patterson G, et al. Xanthine oxidase as a source of free radical damage in myocardial ischemia. J Moll Cell Cardiol 1985;17:145-152.

59. Werns SW, Shea MJ, Mitsos SE. Reduction of the size of infarction by allopurinol in the ischemic-reperfused canine heart. Circulation 1986;73:518-524.

60. Reimer KA, Jennings RB. Failure of the xanthine oxidase inhibitor allopurinol to limit infarct size after ischemia and reperfusion in dogs. Circulation 1985;71:1069-1075.

61. Werns SW, Grum CM, Ventura A, et al. Effects of allopurinol or oxypurinol on myocardial reperfusion injury (abstr). Circulation 1987;76:IV-97.

62. Matsuki T, Shirato C, Cohen MV, et al. Oxipurinol and allopurinol reduce infarct size in reperfused dog heart without pretreatment (abstr). Physiologist 1987;30:187.

63. Puett DW, Forman MB, Cates CU, et al. Oxypurinol limits myocardial stunning but does not reduce infarct size after reperfusion. Circulation 1987;76:678-686.

64. Kinsman JM, Murry CE, Richard VJ, et al. The xanthine oxidase inhibitor oxypurinol does not limit infarct size in a canine model of 40 minutes of ischemia with reperfusion. $J$ Am Coll Cardiol 1988;12:209-217.

65. Downey JM, Miura T, Eddy LJ, et al. Xanthine oxidase is not a source of free radicals in the ischemic rabbit heart. $J$ Mol Cell Cardiol 1987;19:1053-1060.

66. Godin DV, Bhimji S. Effects of allopurinol on myocardial ischemic injury induced by coronary artery ligation and reperfusion. Biochem Pharmacol 1987;36:2101-2107.

67. Grum CM, Ragsdale RA, Ketai LH, et al. Absence of xanthine oxidase or xanthine dehydrogenase in the rabbit myocardium. Biochem Biophys Res Com 1986;141:1104- 
1108.

68. Grum CM, Ketai LH, Myers CL, et al. Purine efflux after cardiac ischemia: Relevance to allopurinol cardioprotection. Am J Physiol 1987;252:H368-H373.

69. Das DK, Engelman RM, Clement R, et al. Role of xanthine oxidase inhibitor as free radical scavenger: A novel mechanism of action of allopurinol and oxypurinol in myocardial salvage. Biochem Biophys Res Com 1987;148:314-319.

70. Myers CL, Weiss SJ, Kirsh MM, et al. Effects of supplementing hypothermic crystalloid cardioplegic solution with catalase, superoxide dismutase, allopurinol, or deferoxamine on functional recovery of globally ischemic and reperfused isolated hearts. $J$ Thorac Cardiovasc Surg 1986; 91:281-289.

71. Peterson DA, Kelly B, Gerrard JM. Allopurinol can act as an electron transfer agent. Is this relevant during reperfusion injury? Biochem Biophys Res Com 1986;137:76-79.

72. Moorhouse PC, Grootveld M, Halliwell B, et al. Allopurinol and oxypurinol are hydroxyl radical scavengers. FEBS Lett
1987;213:23-28.

73. Hoey BM, Butler J, Halliwell B. On the specificity of allopurinol and oxypurinol as inhibitors of xanthine oxidase. A pulse radiolysis determination of rate constants for reaction of allopurinol and oxypurinol with hydroxyl radicals. Free Rad Res Comms 1988;4:259-263.

74. Zimmerman BJ, Parks DA, Grisham MB, et al. Allopurinol does not enhance antitoxidant properties of extracellular fluid. Am $J$ Physiol 1988;255H202-H206.

75. Watts RWE, Watts JEM, Seegmiller JE. Xanthine oxidase activity in human tissues and its inhibition by allopurinol. $J$ Lab Clin Med 1965;66:688-697.

76. Eddy LJ, Stewart JR, Jones HP, et al. The free radical producing enzyme, xanthine oxidase, is undetectable in human hearts. Am J Physiol 1987;253:H709-H711.

77. Jarasch E-D, Bruder G, Heid HW. Significance of xanthine oxidase in capillary endothelial cells. Acta Physiol Scand 1986;548:39-46. 